\title{
Low temperature scintillation properties of $\mathrm{Ga}_{2} \mathrm{O}_{3}$
}

Cite as: Appl. Phys. Lett. 115, 081103 (2019); https://doi.org/10.1063/1.5119130

Submitted: 09 July 2019 . Accepted: 02 August 2019 . Published Online: 20 August 2019

V. B. Mykhaylyk (D) H. Kraus, V. Kapustianyk, and M. Rudko

\section{COLLECTIONS}

EP This paper was selected as an Editor's Pick
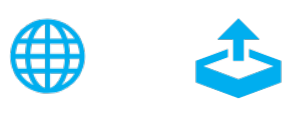

\section{ARTICLES YOU MAY BE INTERESTED IN}

A review of $\mathrm{Ga}_{2} \mathrm{O}_{3}$ materials, processing, and devices

Applied Physics Reviews 5, 011301 (2018); https://doi.org/10.1063/1.5006941

Temperature-dependence of $\mathrm{X}$-ray excited luminescence of $\beta-\mathrm{Ga}_{2} \mathrm{O}_{3}$ single crystals Applied Physics Letters 115, 071904 (2019); https://doi.org/10.1063/1.5110535

Perspective: $\mathrm{Ga}_{2} \mathrm{O}_{3}$ for ultra-high power rectifiers and MOSFETS

Journal of Applied Physics 124, 220901 (2018); https://doi.org/10.1063/1.5062841

\section{Lock-in Amplifiers up to $600 \mathrm{MHz}$}
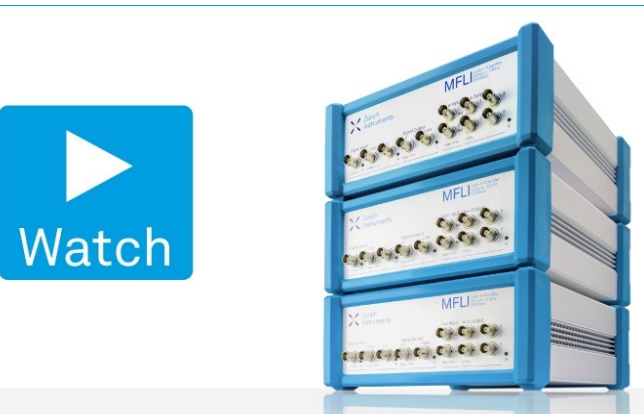


\title{
Low temperature scintillation properties of $\mathrm{Ga}_{2} \mathrm{O}_{3}$ (ㄹ
}

\author{
Cite as: Appl. Phys. Lett. 115, 081103 (2019); doi: 10.1063/1.5119130 \\ Submitted: 9 July 2019 . Accepted: 2 August 2019 . \\ Published Online: 20 August 2019

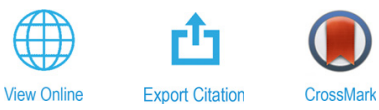

\section{B. Mykhaylyk, ${ }^{1, a)}$ (D) H. Kraus, ${ }^{2}$ V. Kapustianyk, ${ }^{3}$ and M. Rudko ${ }^{3}$}

\begin{abstract}
AFFILIATIONS
'Diamond Light Source, Harwell Campus, Didcot, OX11 ODE, United Kingdom

${ }^{2}$ Department of Physics, University of Oxford, Denys Wilkinson Building, Keble Road, Oxford, OX1 3RH, United Kingdom

${ }^{3}$ Scientific-Technical and Educational Centre of Low Temperature Studies, I. Franko National University of Lviv, 50 Dragomanova Str., 79005, Lviv, Ukraine
\end{abstract}

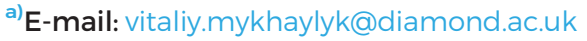

\begin{abstract}
Gallium oxide has recently been identified as a promising scintillator. To assess its potential as a detector material for ionizing radiation at low temperatures, we measured the luminescence and scintillation properties of an undoped $\mathrm{Ga}_{2} \mathrm{O}_{3}$ crystal over the 7-295 K temperature range. The emission of the crystal is due to the radiative decay of self-trapped excitons and donor-acceptor pairs and peaks at a wavelength of $380 \mathrm{~nm}$. The scintillation light output of the undoped $\mathrm{Ga}_{2} \mathrm{O}_{3}$ increases with a decrease in temperature, reaching a maximum value of 19 $300 \pm 2200 \mathrm{ph} / \mathrm{MeV}$ at $50 \mathrm{~K}$. The measured luminescence kinetics has a recombination character with specific decay time $\left(\tau_{0.1}\right)$ increasing from 1 to $1.8 \mu$ s at cooling. Since radiative decay in the crystal competes with nonradiative processes, material optimization could lead to the scintillator achieving a yield of $40800 \mathrm{ph} / \mathrm{MeV}$, a figure considered to be an upper limit.
\end{abstract}

Published under license by AIP Publishing. https://doi.org/10.1063/1.5119130

X-rays, $\gamma$-quanta, and particles (electrons, neutrons, alpha) are extensively used for scientific explorations and in many applications related to medicine, industry, security, and environmental monitoring. The scintillation method, involving the conversion of deposited energy to light, is widely used for the detection of high-energy ionizing radiation. The critical advantage of a scintillation detector is its nonreliance on the extraction of charged particles from the material. Scintillation light can be detected directly from the bulk of the crystal absorber. Here, it is generated by radiative decay of excited particles with a very fast response, characteristic of exciton emission. Therefore, the development of more efficient materials for scintillation detection remains an important topic in relevant areas of modern scientific research. The topical review ${ }^{1}$ gives a good overview on the subject.

Semiconductor materials are of particular interest as scintillators due to their generally high conversion efficiency which translates directly into enhanced energy resolution. This is caused by the lower bandgap compared with that typical for dielectric scintillators and the higher mobility of free carriers. The light yield per unit of absorbed energy is inversely proportional to the value of the energy gap of a material, ${ }^{2,3}$ so that the decrease in this parameter results in higher scintillation efficiency. Moreover, the high mobility of free electrons and holes in semiconductors ensures their prompt escape from the region of the ionization track, with high probability of nonradiative quenching. ${ }^{4,5}$ This facilitates the transfer of excitation energy to the activators. Consequently, doped semiconductor scintillators, such as $\mathrm{ZnS}-\mathrm{Ag}$, $\mathrm{ZnSe}-\mathrm{Te},{ }^{7}$ and $\mathrm{ZnTe}-\mathrm{O},{ }^{8}$ demonstrate high light yield at room temperature, warranting their application in detectors for high-energy photons and particles. Another example is Ga-doped $\mathrm{ZnO}$, exhibiting high light yield and subnanosecond decay time constant due to exciton emission. ${ }^{9}$ Recently, the scintillation properties of undoped $\mathrm{Ga}_{2} \mathrm{O}_{3}$ have been examined for the first time, and it was shown that the material is a promising scintillator, exhibiting a light yield of $15000 \mathrm{ph} / \mathrm{MeV}$ and a decay time constant of $8 \mathrm{~ns}$ at room temperature. $^{10}$ This triggered further investigations of the material scintillation properties. $^{11,12}$

This finding also prompted us to investigate the performance of this material as cryogenic scintillator, driven by the development of techniques for particle detection at low temperature. The latter is currently motivated by the requirements of experiments in fundamental physics searching for neutrinoless double beta decay ${ }^{13}$ and dark matter particles. ${ }^{14}$ Aiming to achieve maximum sensitivity, some of these experiments use cryogenic phonon-scintillation detectors that feature both high-energy resolution and event discrimination capability. ${ }^{15-17}$ The principal advantage of this technique lies in a significant (by few 
orders of magnitude) reduction of contributions from radioactive background. Recent achievement is demonstrated by the latest results from cryogenic experiments searching for neutrinoless double beta decay ${ }^{18}$ and dark matter particles. ${ }^{19}$ There is continuing interest in furthering this technique and finding new scintillation materials suitable for low temperature application and is an important aspect of related research. ${ }^{3,15,20}$ Furthermore, this activity creates a foundation for the exploration of new concepts and applications, of which the most appealing is harnessing the time-of-flight detection method for positron emission tomography using fast and bright cryogenic scintillators. $^{21,22}$

In this work, we carried out measurements of X-ray luminescence, decay kinetics, and scintillation light output of undoped $\mathrm{Ga}_{2} \mathrm{O}_{3}$ as a function of temperature. By exploring and analyzing the temperature dependence of these properties, we extended our knowledge of the material and assessed the prospect of $\mathrm{Ga}_{2} \mathrm{O}_{3}$ for application as cryogenic scintillation detector. These studies provide additional valuable information about the processes that transform absorbed energy into scintillation, which also improves the general understanding of the scintillation mechanism in solids at fundamental levels.

The sample of undoped $\beta-\mathrm{Ga}_{2} \mathrm{O}_{3}$, used in this study, was obtained from Tamura Co. (Japan). For the luminescence measurements, the sample was placed into a closed-cycle He cryostat, equipped with a DE-202A cryocooler (Advanced Research Systems) and Cryocon 32 (Cryogenic Control Systems Inc.) temperature regulator. The steady-state X-ray luminescence was excited by a URS-55A X-ray tube with a $\mathrm{Cu}$-anticathode tube operating at $55 \mathrm{kV}$ and $10 \mathrm{~mA}$. The emission spectra were recorded using an MDR-12 spectrograph with a spectral resolution of $1 \mathrm{~nm}$ and a Hamamatsu H9305 photomultiplier module, sensitive over a $200-700 \mathrm{~nm}$ wavelength range.

For measurements of scintillation characteristics, the crystal was placed in a helium constant flow cryostat and excited by $\alpha$-particles from an ${ }^{241} \mathrm{Am}$ source. The measurements and analyses were carried out using the multiphoton counting technique. ${ }^{23}$ The signal, detected by a multialkali photomultiplier model $9124 \mathrm{~A}$ (Electron Tubes Enterprises, Ruislip, UK), was digitized by a fast analogue-to-digital converter (ADC) with a $5 \mathrm{~ns}$ sampling interval. This allows resolving individual photons and recording single photon signals (SPSs). The technique enables studies of scintillation processes with decay time constants in the range of $10^{-6}-10^{-3} \mathrm{~s}$. Spanning such a wide range when changing the operating temperature is quite common in many scintillation materials. Furthermore, it allows measuring both decay time and light yield characteristics of scintillators in a single experiment. It is therefore well suited for the investigation of temperature-dependent scintillator properties as documented in various publications. ${ }^{3,23,24}$

The most common $\beta$-polymorph of gallium oxide has a monoclinic crystal structure (space group $\mathrm{C} 2 / \mathrm{m}$ ) with lattice constants $\mathrm{a}=12.214 \AA, \mathrm{b}=3.037 \AA, \mathrm{c}=5.798 \AA, \alpha=90^{\circ}, \beta=103.83^{\circ}$, $\gamma=90^{\circ}$. ${ }^{25}$ The crystal unit cell contains $\mathrm{GaO}_{6}$ octahedral and $\mathrm{GaO}_{4}$ tetrahedral chains aligned along the b-axis. Oxygen atoms are located in three crystallographically different positions and form a distorted cubic closed packed array. It is a wideband semiconductor with a bandgap energy $E_{g}=4.85 \mathrm{eV}$, a density of $5.95 \mathrm{~g} / \mathrm{cm}^{3}$, and a melting point of $1795^{\circ} \mathrm{C}$.

Under steady-state X-ray excitation, $\mathrm{Ga}_{2} \mathrm{O}_{3}$ exhibits a broad emission band in the UV-green region of emission spectrum that gradually increases in intensity with cooling the crystal (see Fig. 1).

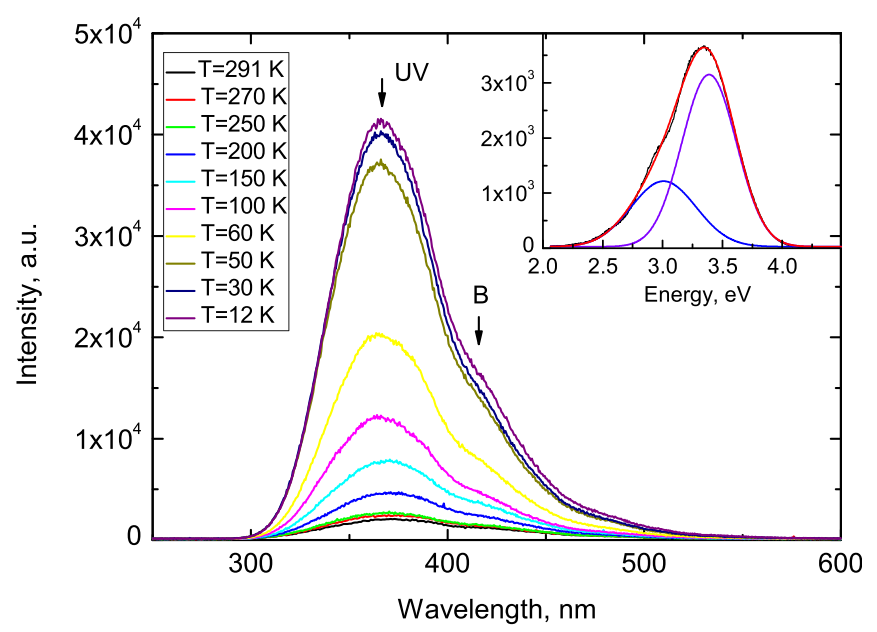

FIG. 1. X-ray luminescence spectra of $\mathrm{Ga}_{2} \mathrm{O}_{3}$ measured at different temperatures. Two emission bands are marked as UV and $\mathrm{B}$. The inset shows the emission spectrum at $\mathrm{T}=12 \mathrm{~K}$ as a function of energy, decomposed into two Gaussians peaking at $3.01 \mathrm{eV}$ (blue emission) and $3.38 \mathrm{eV}$ (UV emission), respectively.

The emission band peaking at $380 \mathrm{~nm}$ has a complex character, which is a characteristic for a system where transitions occur within different emission centers. This is demonstrated in the inset of Figure 1, where the emission spectrum measured at a temperature of $12 \mathrm{~K}$ is plotted as a function of photon energy.

The spectrum can be represented as a sum of two Gaussians at 3.01 and $3.38 \mathrm{eV}$, indicating that at least two types of emission centers exist in the crystal under study. Such a composite structure of luminescence spectra is a characteristic feature of gallium oxide. Previous studies of luminescence properties of undoped $\mathrm{Ga}_{2} \mathrm{O}_{3}$ reported at least three strongly overlapping emission bands in UV, blue, and green parts of the emission spectra. ${ }^{27-30}$ The UV band is generally independent of specific impurities and thus is assigned to the emission of self-trapped excitons. This emission typically manifests fast kinetics in the nanosecond range. The blue and green bands are attributed to transitions involving deep donors and acceptors. Electrons recombining with holes at the defect sites are the origin of recombination luminescence with long decay kinetics (microsecond range). The formation energy of different types of intrinsic defects in $\beta-\mathrm{Ga}_{2} \mathrm{O}_{3}$ was analyzed in a recent theoretical work. ${ }^{31}$ The results of that study confirmed that blue emission is due to complexes of oxygen and gallium vacancies $\left(\mathrm{V}_{\mathrm{o}}+\mathrm{V}_{\mathrm{Ga}}\right)$.

Further, we studied the scintillation properties of undoped $\mathrm{Ga}_{2} \mathrm{O}_{3}$ over the temperature range of $7-295 \mathrm{~K}$. The crystal exhibits a good scintillation response allowing to detect a peak due to $\alpha$-particles from an ${ }^{241} \mathrm{Am}$ source depositing their kinetic energy in the scintillator. The resulting pulse height spectra of the crystal at different temperatures are displayed in Fig. 2. Given that scintillation light yield is proportional to the position of this peak, the variation of the light output of the crystal under investigation with temperature can be traced by monitoring the change in the peak position. The temperature dependence of the light output for the undoped $\mathrm{Ga}_{2} \mathrm{O}_{3}$ derived in this way is shown in Fig. 3. With cooling the crystal, the peak shifts toward higher amplitudes, indicating a steady rise of the scintillation light output. This trend is observed until the temperature is lowered to $50 \mathrm{~K}$, 

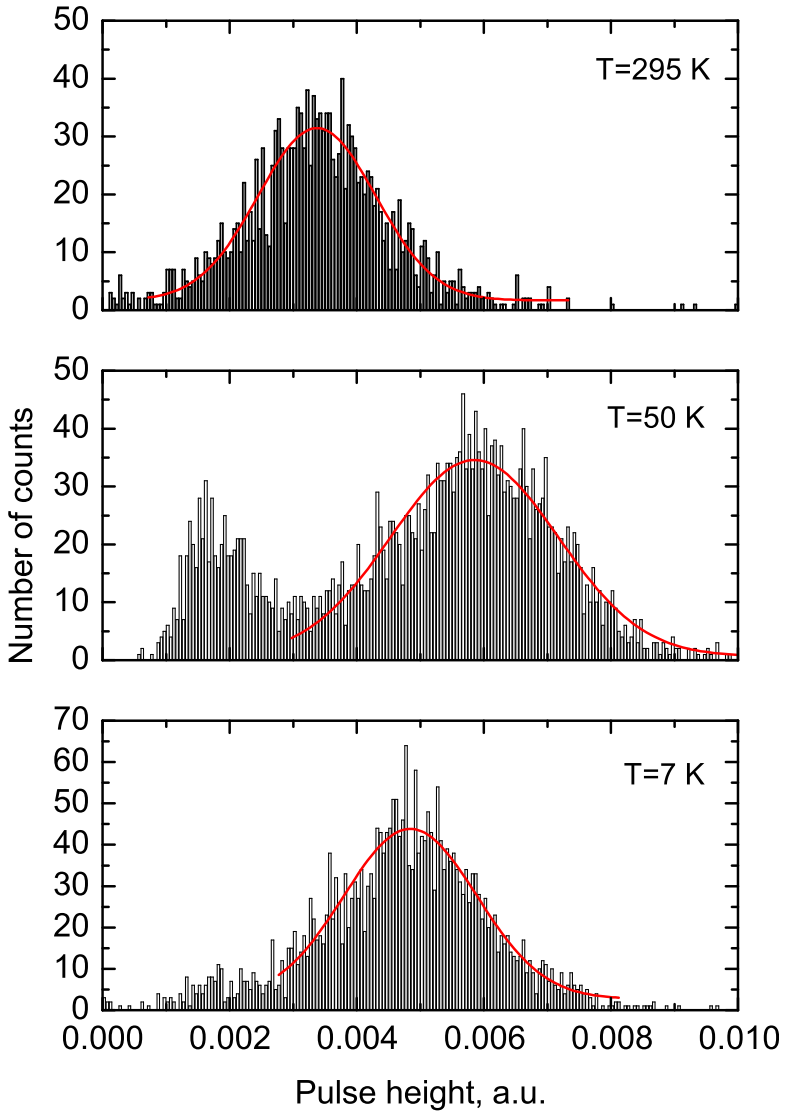

FIG. 2. Pulse height spectra of $\mathrm{Ga}_{2} \mathrm{O}_{3}$ at $\mathrm{T}=7,50$, and $295 \mathrm{~K}$, measured at the excitation with $\alpha$-particles from ${ }^{241} \mathrm{Am}$. The curve lines show the Gaussian fitting the spectra. The feature observed at the low-energy side of the $\alpha$-peak at low temperatures is due to the gradual cut-off of background by a trigger affected by the baseline nose.

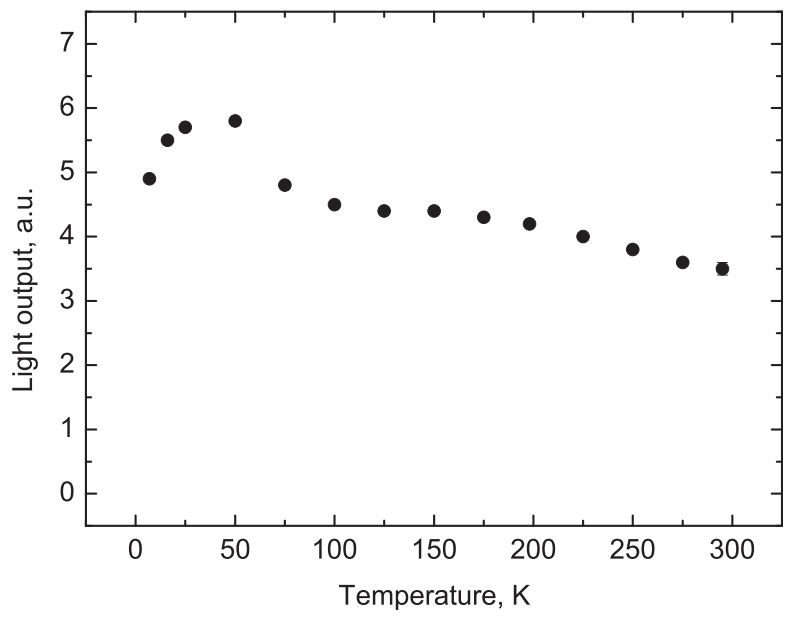

FIG. 3. Scintillation light output of $\mathrm{Ga}_{2} \mathrm{O}_{3}$ as a function of temperature, measured for the excitation with $\alpha$-particles from ${ }^{241} \mathrm{Am}$. below which the scintillation light output starts to decrease, as shown in Fig. 3. At $7 \mathrm{~K}$, the measured scintillation light output of the $\mathrm{Ga}_{2} \mathrm{O}_{3}$ crystal reduces to about $80 \%$ of its maximum value. It is worthwhile noting that the decrease in scintillation light yield at very low temperature has been observed in some other scintillators. One explanation of this feature is a capture of excited carriers by shallow traps ${ }^{32,33}$ that cause long-lasting afterglow. Due to charge trapping, the excitation energy is not anymore released during the actual scintillation event but over a much longer period of time $\left(10^{-3}-1 \mathrm{~s}\right)$ as phosphorescence signal and the corresponding fraction of excitation cannot therefore be detected, thus resulting in a decrease in the scintillation output.

Figure 4 shows the scintillation decay curves of undoped $\mathrm{Ga}_{2} \mathrm{O}_{3}$ monitored at different temperatures. We measured scintillation decay curves in the integral regime, capturing the entire emission spectrum of the scintillator, but due to the limited timing resolution of the measuring technique $(0.1 \mu \mathrm{s})$, the nanosecond component reported by Yanagida et al. ${ }^{10}$ cannot be identified. The existence of several types of emission centers with different types of decay kinetics results in a complex nonexponential decay that is a characteristic feature of recombination luminescence. ${ }^{34}$ This complexity of the scintillation process imposes certain limitations on the analysis of the decay kinetics. More specifically, the fitting of the scintillation decay curves using a linear combination of exponential functions is merely a mathematical way of representing the experimental results. In such a case, it is not possible to relate the fitting parameters directly to the physical quantities that describe the specific emission processes. Therefore, in such a case, more practical quantitative characteristic of the decay is a time when the initial intensity decreases by an order of magnitude $\left(\tau_{0.1}\right)$. The temperature variation of the $\tau_{0.1}$ in the crystals under investigation is shown in the inset of Fig. 4. The shape of the scintillation curves is largely unaffected by temperature; the only noticeable change-the emergence of a long component-is observed at $\mathrm{T}<60 \mathrm{~K}$. We attribute this component to the abovementioned process of recombination of carriers released from the shallow traps. Otherwise, this behavior is consistent with what has been seen in other semiconductor scintillators. $^{6,35}$

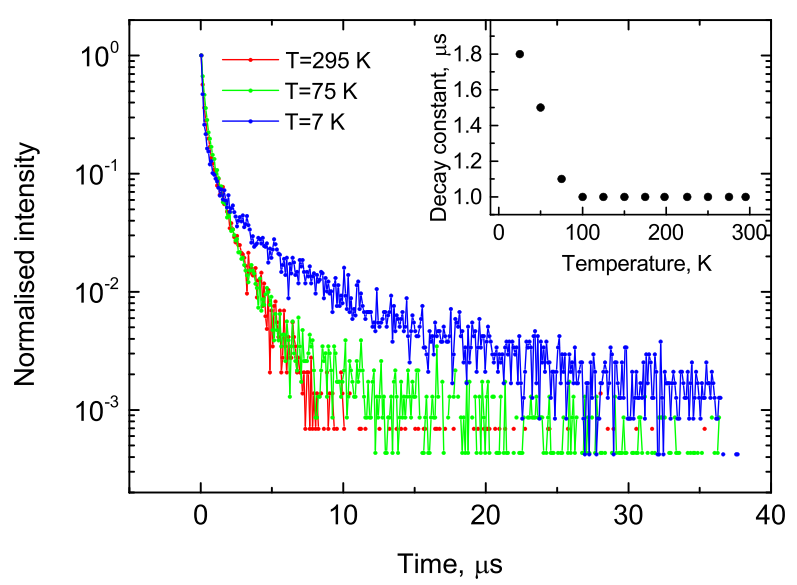

FIG. 4. Normalized scintillation decay curves of $\mathrm{Ga}_{2} \mathrm{O}_{3}$ at $\mathrm{T}=7,75$, and $295 \mathrm{~K}$ measured at the excitation with $\alpha$-particles from ${ }^{241} \mathrm{Am}$. The inset shows the temperature dependence of the decay constant $\tau_{0.1}$. 
To assess the performance of the $\mathrm{Ga}_{2} \mathrm{O}_{3}$ as the scintillator, it is essential to quantify the light yield of the material. First, we calculated the theoretical limit for the absolute light yield of the $\mathrm{Ga}_{2} \mathrm{O}_{3}$ scintillator using the semiempirical approach developed by Lempincki et al. ${ }^{2}$ and later refined in Ref. 3. In this case, the energy transfer efficiency and luminescence quantum efficiency are assumed to be equal to 1, bringing about the following equation for the absolute light yield of a scintillator:

$$
L Y=\frac{10^{6}}{2.35 E_{g}}\left[1+0.158 \times 10^{4}\left\{\frac{1}{\varepsilon_{\infty}}-\frac{1}{\varepsilon_{0}}\right\} \frac{\left(h \nu_{L O}\right)^{\frac{3}{2}}}{1.5 E_{g}}\right]^{-1}(\mathrm{ph} / \mathrm{MeV}) .
$$

Here, $\varepsilon_{0}=11.2$ and $\varepsilon_{\infty}=3.8^{26}$ are the static and high-frequency relative permittivities, respectively, of the material, and $h \nu_{L O}=99 \mathrm{meV}^{36}$ is the maximum energy of longitudinal optical (LO) phonons. Substituting the numerical values into Eq. (1), we obtained an upper limit for the light yield of $\mathrm{Ga}_{2} \mathrm{O}_{3}$ equal to $40800 \mathrm{ph} / \mathrm{MeV}$.

We also measured the light yield of $\mathrm{Ga}_{2} \mathrm{O}_{3}$ experimentally. Typically, the light yield is derived by comparing the scintillation response of the crystal under study with that of a reference scintillator measured under identical experimental conditions. This approach assumes that the light collection efficiency of the experimental setup used for the measurements is a constant parameter determined by geometrical factors that are identical. In this case, we used as a reference an $\mathrm{Lu}_{2(x-1)} \mathrm{Y}_{2 x} \mathrm{SiO}_{4}$-Ce (LYSO-Ce) scintillator, known for its high light yield (34 $000 \mathrm{ph} / \mathrm{MeV}$ ) and fast decay time (33 ns), both changing only insignificantly with cooling. ${ }^{37,38}$ This scintillator emits in the same spectral range as $\mathrm{Ga}_{2} \mathrm{O}_{3}$, which is beneficial for reducing the errors arising from the uncertainty of calculating the emission-weighted detector efficiency $\varepsilon_{\lambda}{ }^{39,40}$ This parameter accounts for the difference in the spectral sensitivity of the detector and was calculated from the measured X-ray luminescence spectra of the crystals and the known quantum efficiency of the 9124A photomultiplier.

The value of $\varepsilon_{\lambda}$ was found to be 0.27 and 0.25 for $\mathrm{Ga}_{2} \mathrm{O}_{3}$ and LYSO-Ce, respectively. Under the aforementioned assumption of identical light collection efficiency, the light output measured in the experiment is proportional to the two variables, i.e., absolute light yield (LY) and emission-weighted detector sensitivity: ${ }^{39,40} N_{m} \sim L Y \times \varepsilon_{\lambda}$. Thus, from the measurements obtained with the two scintillators, we can estimate the light yield of $\mathrm{Ga}_{2} \mathrm{O}_{3}$ using

$$
L Y_{G a 2 O 3}=L Y_{L Y S O} \times \frac{N_{G a 2 O 3}}{N_{L Y S O}} \times \frac{\varepsilon_{\lambda, L Y S O}}{\varepsilon_{\lambda, G a 2 O 3}} .
$$

Taking into account a typical value of the absolute light yield of LYSO-Ce, we determined that the light yield of $\mathrm{Ga}_{2} \mathrm{O}_{3}$ is equal to 11 $600 \pm 1600 \mathrm{ph} / \mathrm{MeV}$ at $295 \mathrm{~K}$, reaching a maximum value of 19 $300 \pm 2200 \mathrm{ph} / \mathrm{MeV}$ at $50 \mathrm{~K}$. The error of this evaluation comes mainly from the uncertainty in the position of the peak that corresponds to $\alpha$-events in the measured scintillators. This finding evidences that undoped $\beta-\mathrm{Ga}_{2} \mathrm{O}_{3}$ can be used for scintillation detection over a wide temperature range. Comparison of the measured light yield with the theoretical prediction also shows that this parameter for the measured crystal is only half of what it can be, and hence gallium oxide has a significant potential for further improvement of its scintillation properties.
In this work, we report the scintillation and luminescence properties of $\beta-\mathrm{Ga}_{2} \mathrm{O}_{3}$ measured as a function of temperature down to $7 \mathrm{~K}$. Two emission bands observed in the UV and blue spectral ranges are assigned to the radiative recombination of self-trapped excitons and donor-acceptor pairs, respectively. The scintillation light output of the crystal increases progressively with a decrease in temperature, reaching a maximum value of $19300 \pm 2200 \mathrm{ph} / \mathrm{MeV}$ at $50 \mathrm{~K}$, from where it reduces by $30 \%$ at further cooling to $7 \mathrm{~K}$. It is found that at room temperature, undoped $\mathrm{Ga}_{2} \mathrm{O}_{3}$ exhibits about one-third of the light output of a commercial LYSO-Ce scintillator. The detection efficiency of the crystal is lower in comparison with the champions in the field, but taking into consideration the fact that intrinsic defects play a major role in the emission of gallium oxide, we envisage that the substantial improvement of the scintillation properties is likely to occur through material doping and optimization of production technology.

\section{REFERENCES}

${ }^{1}$ C. Dujardin, E. Auffray, E. Bourret-Courchesne, P. Dorenbos, P. Lecoq, A. N. Vasil'ev, A. Yoshikawa, and R.-Y. Zhu, "Needs, trends and advances in inorganic scintillators," IEEE Transitions Nucl. Sci. 65, 1977-1997 (2018).

$2^{2}$. Lempicki, A. J. Wojtowicz, and E. Berman, "Fundamental limits of scintillator performance," Nucl. Instrum. Methods Phys. Res., Sect. A 333, 304-311 (1993).

${ }^{3}$ V. B. Mikahilik and H. Kraus, "Performance of scintillation materials at cryogenic temperatures,” Phys. Status Solidi B 247, 1583-1599 (2010).

${ }^{4}$ X. Lu, Q. Li, G. A. Bizarri, K. Yang, M. R. Mayhugh, P. R. Menge, and R. T. Williams, "Coupled rate and transport equations modeling proportionality of light yield in high-energy electron tracks: CsI at $295 \mathrm{~K}$ and $100 \mathrm{~K}$; CsI:Tl at 295 K," Phys. Rev. B 92, 115207 (2015).

${ }^{5}$ M. P. Prange, Y. L. Xie, L. W. Campbell, F. Gao, and S. Kerisit, "Monte Carlo simulation of electron thermalization in scintillator materials: Implications for scintillator nonproportionality," J. Appl. Phys. 122, 234504 (2017).

${ }^{6}$ V. B. Mikhailik, S. Henry, M. Horn, H. Kraus, A. Lynch, and M. Pipe, "Investigation of luminescence and scintillation properties of a $\mathrm{ZnS}-\mathrm{Ag} / 6 \mathrm{LiF}$ scintillator in the 7-295K temperature range," J. Lumin. 134, 63-66 (2013).

${ }^{7}$ V. Ryzhikov, N. Starzhinskiy, L. Gal'chinetskii, P. Gashin, D. Kozin, and E. Danshin, "New semiconductor scintillators $\mathrm{ZnSe}(\mathrm{Te}, \mathrm{O})$ and integrated radiation detectors based thereon," IEEE Trans. Nucl. Sci. 48, 356-359 (2001).

${ }^{8}$ Z. T. Kang, C. J. Summers, H. Menkara, B. K. Wagner, R. Durst, Y. Diawara, G. Mednikova, and T. Thorson, "ZnTe:O phosphor development for X-ray imaging applications,” Appl. Phys. Lett. 88, 111904 (2006).

${ }^{9}$ Y. Fujimoto, T. Yanagida, H. Sekiwa, Y. Yokota, V. Chani, and A. Yoshikawa, "Scintillation characteristic of In,Ga-doped $\mathrm{ZnO}$ thin films with different dopant concentrations," Jpn. J. Appl. Phys., Part 1 50, 01BG04 (2011).

${ }^{10}$ T. Yanagida, G. Okada, T. Kato, D. Nakauchi, and S. Yanagida, "Fast and high light yield scintillation in the Ga2O3 semiconductor material," Appl. Phys. Express 9, 042601 (2016).

${ }^{11}$ N. He, H. Tang, B. Liu, Z. Zhu, Q. Li, C. Guo, M. Gua, J. Xu, J. Liu, M. Xu et al., "Ultra-fast scintillation properties of $\beta$-Ga2O3 single crystals grown by floating zone method,” Nucl. Instrum. Methods Phys. Res., Sect. A 888, 9-12 (2018).

${ }^{12}$ W. Drozdowski, M. Makowski, M. E. Witkowski, A. J. Wojtowicz, Z. Galazka, K. Irmscher, and R. Schewski, " $\beta-\mathrm{Ga} 2 \mathrm{O} 3: \mathrm{Ce}$ as a fast scintillator: An unclear role of cerium,” Radiat. Meas. 121, 49-53 (2019).

${ }^{13}$ J. D. Vergados, H. Ejiri, and F. Šimkovic, "Neutrinoless double beta decay and neutrino mass," Int. J. Mod. Phys. 25, 1630007 (2016).

${ }^{14}$ A. H. G. Peter, V. Gluscevic, A. M. Green, B. J. Kavanagh, and S. K. Lee, "WIMP physics with ensembles of direct-detection experiments," Phys. Dark Universe 5-6, 45-74 (2014).

${ }^{15}$ V. B. Mikhailik and H. Kraus, "Cryogenic scintillators in searches for extremely rare events,” J. Phys. D: Appl. Phys. 39, 1181-1191 (2006).

${ }^{16}$ R. F. Lang, G. Angloher, M. Bauer, I. Bavykina, A. Bento, A. Brown, C. Bucci, C. Ciemniak, C. Coppi, G. Deuter et al., "Discrimination of recoil background in scintillating calorimeters," Astropart. Phys. 33, 60-64 (2010). 
${ }^{17}$ D. Poda and A. Giuliani, "Low background techniques in bolometers for double-beta decay search,” Int. J. Mod. Phys. 32, 1743012 (2017).

${ }^{18}$ O. Azzolini, M. T. Barrera, J. W. Beeman, F. Bellini, M. Beretta, M. Biassoni, C. Brofferio, C. Bucci, L. Canonica, S. Capelli et al., "First result on the neutrinoless double- $\beta$ decay of 82 Se with CUPID-0," Phys. Rev. Lett. 120, 232502 (2018).

${ }^{19}$ G. Angloher, P. Bauer, A. Bento, E. Bertoldo, C. Bucci, L. Canonica, A. D'Addabbo, X. Defay, S. Di Lorenzo, A. Erb et al., "Limits on dark matter effective field theory parameters with CRESST-II," Eur. Phys. J. C 79, 43 (2019).

${ }^{20}$ S. Derenzo, E. Bourret, S. Hanrahan, and G. Bizarri, "Cryogenic scintillation properties of $\mathrm{n}$-type GaAs for the direct detection of $\mathrm{MeV} / \mathrm{c} 2$ dark matter," J. Appl. Phys. 123, 114501 (2018).

${ }^{21}$ S. E. Derenzo, E. Bourret-Courshesne, G. Bizarri, and A. Canning, "Bright and ultra-fast scintillation from a semiconductor?," Nucl. Instrum. Methods Phys. Res., Sect. A 805, 36-40 (2016).

${ }^{22}$ V. B. Mykhaylyk, H. Kraus, and M. Saliba, "Bright and fast scintillation of organolead perovskite MAPbBr3 at low temperatures," Mater. Horiz. (published online 2019).

${ }^{23}$ V. B. Mikhailik and H. Kraus, "Development of techniques for characterisation of scintillation materials for cryogenic application," Radiat. Meas. 49, 7-12 (2013).

${ }^{24}$ V. B. Mikhailik and H. Kraus, "Scintillators for cryogenic application: State-ofart," J. Phys. Stud. 14, 4201-4206 (2010).

${ }^{25} \mathrm{~J}$. Ahman, G. Svensson, and J. Albertsson, "A reinvestigation of $\beta$-gallium oxide," Acta Crystallogr., Sect. C 52, 1336-1338 (1996).

${ }^{26}$ S. I. Stepanov, V. I. Nikolaev, V. E. Bougrov, and A. E. Romanov, "Gallium oxide: Properties and applications-A review,” Rev. Adv. Mater. Sci. 44, 63-86 (2016).

${ }^{27} \mathrm{G}$. Blasse and A. Brill, "Some observations on the luminescence of b-Ga2O3," J. Phys. Chem. Solids 31, 707-711 (1970).

${ }^{28}$ V. I. Vasil'tsiv, Y. M. Zakharko, and Y. I. Rym, "On the nature of blue and green luminescence bands of beta-GA2O3," Ukrainskyi Fizychnyi Zh. 33, 1320-1324 (1988).

${ }^{29} \mathrm{~L}$. Binet and J. Gourier, "Origin of the blue luminescence of b b-Ga2O3," J. Phys. Chem. Solids 59, 1241-1249 (1998).
${ }^{30}$ T. Onuma, S. Fujioka, T. Yamaguchi, M. Higashiwaki, K. Sasaki, T. Masui, and T. Honda, "Correlation between blue luminescence intensity and resistivity in $\beta$-Ga2O3 single crystals,” Appl. Phys. Lett. 103, 041910 (2013).

${ }^{31}$ Q. D. Ho, T. Frauenheim, and P. Deák, "Origin of photoluminescence in $\beta$ Ga2O3," Phys. Rev. B 97, 115163 (2018).

${ }^{32}$ F. A. Danevich, B. V. Grinyov, S. Henry, M. B. Kosmyna, H. Kraus, N. Krutyak, V. M. Kudovbenko, V. B. Mikhailik, L. L. Nagornaya, B. P. Nazarenko et al., "Feasibility study of PbWO4 and $\mathrm{PbMoO} 4$ crystal scintillators for cryogenic rare events experiments," Nucl. Instrum. Methods Phys. Res., Sect. A 622, 608-613 (2010).

${ }^{33}$ L. L. Nagornaya, F. A. Danevich, A. M. Dubovik, B. V. Grinyov, S. Henry, V. Kapustyanyk, H. Kraus, D. V. Poda, V. M. Kudovbenko, V. B. Mikhailik et al., "Tungstate and molybdate scintillators to search for Dark Matter and double beta decay,” IEEE Trans. Nucl. Sci. 56, 2513-2518 (2009).

${ }^{34}$ A. N. Vasil'ev and A. V. Gektin, "Multiscale approach to estimation of scintillation characteristics," IEEE Trans. Nucl. Sci. 61, 235-245 (2014).

${ }^{35}$ V. B. Mikhailik, S. Galkin, H. Kraus, V. Mokina, A. Hrytsak, V. Kapustianyk, M. Panasiuk, M. Rudko, and V. Rudyk, "ZnTe cryogenic scintillator," J. Lummin. 188, 600-603 (2017).

${ }^{36}$ T. Onuma, S. Saito, K. Sasaki, K. Goto, T. Masui, T. Yamaguchi, T. Honda, A. Kuramata, and M. Higashiwaki, "Temperature-dependent exciton resonance energies and their correlation with IR-active optical phonon modes in $\beta$ Ga2O3 single crystals,” Appl. Phys. Lett. 108, 101904 (2016).

${ }^{37}$ D. N. ter Weele, D. R. Schaart, and P. Dorenboss, "Comparative study of codoped and non co-doped LSO:Ce and LYSO:Ce scintillators for TOF-PET," IEEE Trans. Nucl. Sci. 62, 727-731 (2015).

${ }^{38}$ S. Blahuta, A. Bessière, B. Viana, P. Dorenbos, and V. Ouspenski, "Evidence and consequences of $\mathrm{Ce}$ in LYSO:Ce, $\mathrm{Ca}$ and LYSO:Ce, $\mathrm{Mg}$ single crystals for medical imaging applications," IEEE Trans. Nucl. Sci. 60, 3134-3141 (2013).

${ }^{39}$ B. V. Grinyov, V. D. Ryzhikov, O. T. Sidletskiy, G. M. Onyshchenko, and S. N. Galkin, "Absolute light yield determination for LGSO:Ce, CWO, ZnSe:Al, and GSO:Ce crystals,” IEEE Trans. Nucl. Sci. 57, 1236-1240 (2010).

${ }^{40}$ V. Alenkov, O. A. Buzanov, N. Khanbekov, V. N. Kornoukhov, H. Kraus, V. B. Mikhailik, and V. A. Shuvaeva, "Application of the Monte-Carlo refractive index matching (MCRIM) technique to the determination of the absolute light yield of a calcium molybdate scintillator," J. Instrum. 8, P0600 (2013). 